\title{
Le monde comme mystère et comme menace
}

Besoins de transcendance entre étonnement et angoisse

Die Welt als Geheimnis und Bedrohung. Transzendenzbedürfnis zwischen

Staunen und Angst

The World as Mystery and as Menace. The Need for Transcendence between

Astonishment and Anxiety

\section{Sebastian Hüsch}

\section{OpenEdition}

Journals

Édition électronique

URL : http://journals.openedition.org/ceg/2767

DOI : $10.4000 /$ ceg. 2767

ISSN : 2605-8359

\section{Éditeur}

Presses Universitaires de Provence

Édition imprimée

Date de publication : 26 avril 2018

Pagination : 59-70

ISBN : 979-10-320-0160-8

ISSN : 0751-4239

\section{Référence électronique}

Sebastian Hüsch, «Le monde comme mystère et comme menace », Cahiers d'Études Germaniques [En ligne], 74 | 2018, mis en ligne le 26 octobre 2019, consulté le 26 novembre 2020. URL : http:// journals.openedition.org/ceg/2767 ; DOI : https://doi.org/10.4000/ceg.2767 


\title{
Le monde comme mystère et comme menace Besoins de transcendance entre étonnement et angoisse
}

\author{
Sebastian Hüsch \\ Aix Marseille Univ, ÉCHANGES, EA 4236, Aix-en-Provence, France
}

Selon la célèbre formule de Platon, c'est l'étonnement qui est à l'origine de la philosophie ${ }^{1}$. C'est certainement une vision plus séduisante que celle selon laquelle la réflexion philosophique naît d'un désarroi existentiel, de l'angoisse, de l'ennui, ou du désespoir comme cela semble être le cas pour nombre de philosophes modernes notamment ${ }^{2}$. Il paraît toutefois convaincant d'admettre une certaine parenté entre l'étonnement et l'angoisse dans la mesure où ces deux réactions au monde se fondent sur le même phénomène: la perception du monde comme à la fois infıniment mystérieux et infıniment menaçant ${ }^{3}$. Que ce soit

1. Voir Platon, Théétète 155d: "S'étonner, voilà un sentiment qui est tout à fait d'un philosophe. La philosophie n'a pas d'autre origine " (trad. M. Narcy, Paris, Flammarion, 1999). Nous retrouvons l'étonnement comme origine de la philosophie également chez Aristote qui note dans sa Métaphysique: "C'est, en effet, l'étonnement qui poussa, comme aujourd'hui, les premiers penseurs aux spéculations philosophiques " (Aristote, Métaphysique A, 2, 982 b 10, trad. Tricot, Paris, Vrin, 1970, p. 17-18). Or, comme le souligne Stefan Matuschek, l'étonnement comme point de départ de la philosophie prend une direction très différente chez Platon et chez Aristote, même si leurs formules de départ semblent très proches. Chez Aristote, l'étonnement est à l'origine de l'enquête scientifique qui permet de trouver les raisons du phénomène qui avait causé l'étonnement: l'explication des causes du phénomène met fin à l'étonnement. Chez Platon, au contraire, l'étonnement philosophique est au début d'une ascension vers les idées et donne lieu à un étonnement encore plus ample (ekplexis). Voir S. Matuschek, Über das Staunen. Eine ideengeschichtliche Analyse, Tübingen, Max Niemeier, 1991, p. 22 sq.

2. On pensera à des auteurs comme Søren Kierkegaard, Martin Heidegger ou encore Albert Camus. Il n'est pas inintéressant dans ce contexte de noter que Georg Simmel, au début du XX $X^{\mathrm{e}}$ siècle, remarque combien il lui semble curieux que les souffrances de l'humanité soient absentes de l'histoire de la philosophie (voir G. Simmel, Fragmente und Aufsätze, München, 1923, p. 17). Cette réflexion est d'ailleurs reprise ensuite par Theodor W. Adorno (voir Th. W. Adorno, Philosophische Terminologie, vol. 2, Frankfurt a. Main, Suhrkamp, 1974, p. 178). Voir à ce sujet également Emil Angehrn, Die Herausforderung des Negativen. Zwischen Sinnverlangen und Sinnentzug, Basel, Schwabe, 2015, p. 31.

3. Cette ambiguïté est d'ailleurs très bien saisie par la célèbre formule de Blaise Pascal : "Le silence éternel de ces espaces infınis m’effraie " (B. Pascal, Pensées, Paris, Le Livre de Poche, 2000, fragment 233, p. 172) qui exprime ainsi cet étonnement fasciné suscité par ces espaces infınis, mais qui ne peut que causer une sensation d'effroi pour l'être qui s'y voit "jeté", comme le dirait Heidegger, et qui ne peut qu'être écrasé par le contraste entre ces espaces infinis et sa propre situation, éphémère et vulnérable. Nous retrouvons cette ambiguïté entre étonnement et effroi explicitée chez Rudolf Otto dans sa définition du numineux qu'il appelle le mysterium tremendum, mais également en utilisant le terme fascinans (voir R. Otto, Das Heilige. Über das 
donc l'angoisse ou l'étonnement qui prévale dans la contemplation du monde, la volonté de mieux se situer dans cet endroit si étonnant et si angoissant mène - presque naturellement - à des questionnements méta-physiques dans la mesure où la réflexion atteint forcément cette limite où les explications "physiques" s'arrêtent. Or, dans le monde (post-)moderne, le terme même de "métaphysique" est souvent utilisé avec le seul objectif de dénoncer celle-ci comme quelque chose qui doit être abandonné, dépassé, surmonté. Les questions métaphysiques, elles, en revanche, restent ${ }^{4}$; et contrairement à ce que prétendent nombre de scientifiques, les sciences dites "dures" ne pourront pas y répondre ${ }^{5}$. Dans le cadre de cette contribution, je voudrais ainsi poser la question de savoir dans quelle mesure - et sous quelle forme - il est encore possible, en ce début du $\mathrm{XXI}^{\mathrm{e}}$ siècle, d'appréhender l'étonnement et la frayeur existentiels en ayant recours à des explications - ou du moins à des questionnements - invoquant la transcendance.

Pour développer cette problématique, je voudrais par la suite interroger deux approches philosophiques récentes quant à leur capacité à proposer un positionnement de l'Homme dans une perspective de transcendance qui soit également compatible avec l'état des connaissances scientifiques et philosophiques actuelles. C'est, d'un côté, l'herméneutique phénoménologique de la transcendance que développe Thomas Rentsch, et, de l'autre, l'approche anthropologique s'appuyant également sur la philosophie du langage de Ernst Tugendhat et portant sur la mystique comme génératrice de sens.

Si Rentsch et Tugendhat partent ainsi de perspectives méthodologiques très différentes, elles se rejoignent notamment par rapport à trois aspects qui me paraissent cruciaux dans le présent contexte. Ainsi, (1) les approches de ces deux auteurs partent de la conviction partagée selon laquelle les enjeux de la transcendance entrent inévitablement dans le champ de vision de l'homme qui s'étonne - ou qui s'angoisse - devant le monde, et elles semblent s'accorder à attribuer un certain besoin de transcendance à l'homme en tant qu'il est homme, besoin auquel la philosophie se doit de répondre. D’un point de vue épistémologique, (2) on peut constater que tant Rentsch que Tugendhat visent à scrupuleusement tenir compte de la contingence insurmontable de la connaissance de l'homme comme toile de fond de toute réflexion sur des questions liées à la

Irrationale in der Idee des Göttlichen und sein Verhältnis zum Rationalen, Breslau, Trewendt \&t Granier, 1917, p. 13 ff.).

4. Dans le sens du pessimisme qu'exprime Max Horkheimer dans une de ses interviews tardives données au magazine allemand Der Spiegel (1976) on pourrait ajouter un 'pour l'instant', dans la mesure où Horkheimer s'attend à une 'évacuation' des questions métaphysiques au fur et à mesure que la raison instrumentale s'impose définitivement (Max Horkheimer, "Was wir 'Sinn' nennen, wird verschwinden ", en ligne: [http://www.spiegel.de/spiegel/print/d-45226214.html], 05/01/1970, consulté le 21/12/2016).

5. On constate d'ailleurs très régulièrement une confusion de niveaux de réflexion qui prend une description des causalités identifiables dans des phénomènes physiques pour une réponse à la question de savoir pourquoi tel ou tel phénomène existe. Voir aussi la réflexion de Rémi Brague sur la différence entre décrire et comprendre (R. Brague, "Faut pas chercher à comprendre' ", dans Philosophiques, vol. 41, N², 2014, p. 359-363, ici p. 360). 
métaphysique et de transcendance ${ }^{6}$. Le dernier aspect fondamental qui unit les approches de Rentsch et de Tugendhat, c'est (3) l'importance qu'elles accordent à la raison humaine dans la possibilité de développer une perspective de transcendance. Autrement dit: pour tous deux le recours à la transcendance n'implique pas inévitablement le basculement dans l'irrationalité, mais ils revendiquent, au contraire, un lien étroit entre la rationalité humaine et les questionnements métaphysiques.

\section{L'herméneutique phénoménologique de la transcendance de Thomas Rentsch}

En ce qui concerne Thomas Rentsch, philosophe allemand inspiré notamment par la phénoménologie de Martin Heidegger et la philosophie du langage de Ludwig Wittgenstein ${ }^{7}$, cet aspect de rationalité est même au cœur de ses réflexions. L'une des préoccupations fondamentales de la philosophie de Rentsch est de dénoncer une interprétation réductionniste et trop restrictive de la raison humaine. Pour Rentsch, ce n'est rien d'autre qu'un préjugé scientiste et positiviste selon lequel les questionnements liés à la transcendance et à la foi seraient forcement irrationalistes et sans signification (sinnlos). Dans une monographie portant le titre succinct et programmatique Gott ("Dieu "), Rentsch se donne pour but de démontrer qu'il n'y a aucune raison d'écarter la réflexion sur la transcendance et sur Dieu des préoccupations philosophiques. Selon lui, ce serait plutôt le contraire: une telle réflexion serait même centrale et fondamentale afin de pouvoir définir et circonscrire ce que la philosophie peut et veut représenter dans le monde actuel ${ }^{8}$. Selon Rentsch, la " dimension de la problématique traditionnelle de Dieu reste encore aujourd'hui " inextricablement liée à la question d'un sens authentique de notre existence ${ }^{9}$ ". Or, Rentsch souligne en même temps avec insistance qu'une réflexion philosophique sur Dieu et la question de la transcendance se doit impérativement d'être à la hauteur des acquis de la philosophie:

6. Les deux approches revendiquent explicitement leur " honnêteté intellectuelle ". Ce concept emprunté à F. Nietzsche et son rapport à la religion est développé dans un volume dirigé par Gerald Hartung et al. (G. Hartung et al., Religiosität und intellektuelle Redlichkeit, Tübingen Mohr Siebeck, 2012) où le rapport de la religion à l'exigence de Redlichkeit est abordé et éclairé sous différentes perspectives. Tugendhat lui-même dédie un chapitre de L'Homme égocentré et la mystique à cette problématique (voir E. Tugendhat, L'Homme égocentré et la mystique : une étude anthropologique, Paris, La Maison des Sciences, 2010, p. 57 sq.).

7. Voir Thomas Rentsch, Heidegger und Wittgenstein. Existential- und Sprachanalysen zu den Grundlagen philosophischer Anthropologie, Klett-Cotta, Stuttgart, 1985.

8. Voir Rentsch, Gott, 2005, p. VIII, où il précise qu'il s'agit de déterminer le « Selbstverständnis der Philosophie in der Gegenwart. "

9. Rentsch, Gott, p. IX (" Die Dimension der traditionellen Gottesproblematik, der Frage nach Gott ist mit der Frage nach einem authentischen Sinn unserer Existenz untrennbar verbunden. "). Cette prise de position illustre d'ailleurs très clairement que pour Rentsch, en deçà d'un rapport à la transcendance, toute existence reste déficitaire par rapport au sens qu'elle pourrait prendre. 
Ein kritisches Philosophieren kann heute nicht mehr hinter die Ontologie- und Metaphysikkritik Kants, die Ontologiekritik Heideggers, die Sprachkritik Wittgensteins sowie die Gesellschafts- und Ideologie- bzw. Kulturkritik Adornos zurückfallen ${ }^{10}$.

Il s'agit donc pour Rentsch de rétablir une compréhension de la raison humaine plus riche et plus nuancée que celle qui se serait imposée, avec l'avènement de la Modernité, dans le monde occidental. Sur cette base, il développe son postulat selon lequel il existe des phénomènes de transcendance qui non seulement ne sont pas irrationnels, mais qui, au contraire, dépendent de la raison, autrement dit, qui ne valent que pour l'homme en tant qu'il est un être doté de raison ${ }^{11}$. Rentsch identifie trois phénomènes de transcendance qui se manifestent uniquement pour et par la raison humaine, à savoir: La transcendance $d u$ monde, la transcendance $d u$ langage et la transcendance $d u$ Soi (Transzendenz des Selbst). En parlant de la transcendance du monde, Rentsch veut renvoyer à ce fait inexplicable et incompréhensible qu'il y $a$ un monde, que le monde est ${ }^{12}$. La transcendance du langage devient saisissable dans cet autre fait tout aussi inexplicable: que nous puissions parler, et que pouvoir parler représente une condition nécessaire pour fonder notre réalité humaine ${ }^{13}$. Enfın, pour expliquer l'idée de la transcendance du Moi, Rentsch précise:

Unsere praktische Möglichkeit der Selbsttranszendenz beruht [...] auf der Unerkennbarkeit unserer selbst bzw. unseres Wesens in einem objektivistischen, abschließbaren Sinne. [...] Was uns wirklich gründet, entzieht sich als Grenze unseres Erkennens aller Vergegenständlichung, Instrumentalisierung und Empirie ${ }^{14}$.

10. Rentsch, Gott, p. 1. À partir de ces réflexions préliminaires, Rentsch propose d'abord une réflexion sur la possibilité de parler de "Dieu " sans s'exposer au reproche de parler de quelque chose qui n'a pas de sens. Avec comme toile de fond la philosophie du langage du Wittgenstein 'post-Tractatus', Rentsch précise qu'il ne faut pas comprendre le discours sur Dieu comme un discours qui porte sur 'les choses dans le monde' dans un sens empirique - donc pas dans le sens d'une " krude Gegenständlichkeit » (Rentsch, Gott, p. 91). Selon Rentsch, il faut comprendre qu'un énoncé qui évoque Dieu a un statut différent, mais ce qui n'implique pas per se qu'il soit dépourvu de sens même si ce dernier reste à être clarifié.

11. C'est sa réflexion sur la notion de 'réalité' qui montre le sens dans lequel Rentsch veut orienter la discussion. Il s'oppose à la compréhension réductionniste du discours scientiste et positiviste du concept de réalité en soulignant que la réalité de l'être humain est bien plus que celle qui concerne la présence d'objets matériels (dans le sens heideggérien de "Vorhandenheit "). Ainsi il définit: "Wirklichkeit ist die ganze, irreduzible Weltwirklichkeit, in deren Seins- und Sinnhorizont wir unsere Selbstverständnisse und Handlungsmöglichkeiten entdecken und entwerfen, um in Freiheit ein gutes und gelingendes Leben bewusst zu führen " (Rentsch, Gott, p. 49).

12. Rentsch, Gott, p. 59 (" Dass die Welt überhaupt ist, dass es überhaupt etwas gibt und nicht nichts - das kann man nicht direkt erfahren und nicht als normale Tatsachenbehauptung mitteilen. ").

13. Voir Rentsch, Gott, p. 67-68: "Für unsere Zwecke einer philosophisch-theologischen Sinngrundreflexion sind wiederum, wie auch schon bei der ontologisch-kosmologischen Transzendenzdimension, keine mystischen Sondererfahrungen und auch keine quasimythologischen Entwürfe nötig, um diese Transzendenz des Logos zu explizieren. Dass - und wie - wir sprechen können, ist eine unerklärliche, uns vorgängige Bedingung der Möglichkeit und Wirklichkeit unserer humanen Welt. "

14. Rentsch, Gott, p. 76. 
Autrement dit: Il nous est impossible de devenir parfaitement transparents à nous-mêmes, il reste toujours quelque chose qui nous reste inaccessible dans notre compréhension de nous-mêmes, quelque chose qui résiste à une compréhension selon la logique de la raison fonctionnaliste basée sur les 'faits empiriques', quelque chose qui nous transcende.

Selon Rentsch, ces trois phénomènes de transcendance sont constitutifs de l'être humain, ils ne représentent rien de moins que "les conditions fondant la possibilité et la réalité de notre monde humain ${ }^{15}$. "S'il est évident que ces phénomènes ne sont pas à comprendre comme des faits empiriques, comme 'objets dans le monde', il est tout aussi indéniable qu'ils ont tout de même une réalité, une réalité fondamentale et constitutive pour l'homme au point que, comme le formule Rentsch, "sans ces phénomènes d'une transcendance absolue aucune immanence n'est possible, ni réelle ni même pensable ${ }^{16}$ ".

Si l'on concède la possibilité d'une conceptualisation de la raison dépassant le cadre restreint de ce qu'on pourrait appeler, avec Horkheimer, la raison instrumentale ${ }^{17}$, comme le propose Rentsch, on doit également concéder que la transcendance en tant que phénomène est à la fois " connaissable, rationnelle (rational) et universelle ${ }^{18}$ ". Si Rentsch revendique ainsi une défınition plus large de la raison que ne le propose la conceptualisation scientisante dominante, il reste en même temps vigilant quant au respect des limites de la raison même dans ce sens élargi; il accorde même un rôle constitutif à ces limites dans la mesure où les phénomènes de transcendance que Rentsch évoque se manifestent précisément dans cette région frontalière où la raison atteint ses limites. Les phénomènes de transcendance renvoient donc ex negativo à une transcendance absolue :

Die Vernunft gelangt durch kritische Selbstreflexion an ihre für sie sinnkonstitutiven Grenzen. Die praktische Einsicht in diese Grenzen führt zur ekstatischen Vernunft, die ihren Transzendenzbezug nicht bloß abspaltet und negiert, sondern kritisch-hermeneutisch und dialektisch einbegreift und praktisch zu verstehen sucht.

Et de conclure ce passage par la réflexion suivante: "Dieu”, c'est le terme éminent (Inbegriff) qui représente cette compréhension ${ }^{19}$."

C'est ainsi dans le contexte de ses réflexions sur les phénomènes de transcendance que Rentsch fait l'emploi "critique" de l'expression "Dieu ". Ce nom lui sert donc pour tenir lieu de et faire référence à cette transcendance absolue que la raison ex-statique peut faire entrer dans son champ de vision en tant que ce qui se soustrait à elle ${ }^{20}$.

15. Rentsch, Gott, p. 67 (" [...] uns vorgängige[n] Bedingung[en] der Möglichkeit und Wirklichkeit unserer humanen Welt "; voir n. 13).

16. Rentsch, Gott, p. 78 (" Ohne diese absolute Transzendenz ist keine Immanenz möglich, wirklich oder nur denkbar ").

17. Voir Max Horkheimer, Eclipse of Reason, New York, Oxford University Press, 1947.

18. Rentsch, Gott, p. 99.

19. Ibid., p. 208.

20. Rentsch se place d'ailleurs explicitement dans la tradition de la théologie négative du néoplatonisme à Wittgenstein (voir ibid., p. 122). 
Avant de passer à Tugendhat, je souhaiterais revenir à l'idée évoquée dans l'introduction. Chez Rentsch, c'est principalement à travers l'étonnement philosophique que la perspective de transcendance entre en jeu. Ses réflexions sont focalisées sur le mystérieux plutôt que sur l'angoissant, le mystérieux qui l'est uniquement parce que nous sommes dotés de raison. Chez Rentsch, la réflexion philosophique est donc incitée à partir de ces phénomènes mystérieux que sont le monde, le Soi, le langage et qui aboutissent à une conversion qui sort l'homme de ce qui est purement immanent, non pas en le niant, mais de sorte que le rapport à l'immanence soit transformé, ou comme le dit Kierkegaard, comme dans une "métamorphose ${ }^{21}$ ". C'est à cela que renvoie Rentsch quand il note:

\footnotetext{
Aus diesem Grund sind auch authentische Bekehrungsprozesse so tiefgreifend und grundstürzend: Wenn wir in ein auf Gott, absoluten Sinn und das Wunder der Schöpfung gegründetes Selbstverständnis eintreten, dann wandelt sich das gesamte Welt- und Selbstverständnis. In gewisser Weise ist nichts mehr wie vorher ${ }^{22}$.
}

Autrement dit, dans la métamorphose qui se produit par l'établissement d'un lien à la transcendance, tout reste le même, mais tout, en même temps, n'est plus comme avant. Et cette possibilité de conversion trouve ses origines dans les facultés de la raison humaine ${ }^{23}$.

\section{Ernst Tugendhat: mystique vs. religion}

Si nous nous tournons maintenant vers Tugendhat, nous sommes également confrontés à une argumentation qui lie étroitement transcendance et sens. Selon Tugendhat, la possibilité d'une perspective de transcendance est la particularité la plus fondamentale qui distingue l'homme des " autres animaux ${ }^{24}$ ", comme il le formule, et qui est liée à la possibilité de pouvoir se projeter dans l'avenir - pour y découvrir, outre un champ de possibles, la finitude de sa propre existence. La projection dans l'avenir génère en quelque sorte une double préoccupation de l'homme, l'une “terre-à-terre", l'autre "métaphysique" :

Les êtres humains se vouent d'une part entièrement à leurs activités et à leurs soucis particuliers, mais ils sont d'autre part inquiétés par la question portant sur ce qu'ils veulent au fond, sur ce qui est décisif dans leur vie ${ }^{25}$.

Or, comme cette deuxième question confronte l'homme avec sa fragilité et sa précarité, l'homme se retrouve dans une situation ambivalente: il est tiraillé

21. Voir Søren Kierkegaard, Entweder/Oder, München, dtv, 2005, p. 840.

22. Rentsch, Gott, p. 113.

23. Il y a, bien sûr, néanmoins un lien avec les affectations négatives comme incitation à la réflexion philosophique qui s'invitent au moins implicitement notamment dans le contexte de la réflexion sur le sens. Le besoin de sens qui est comblé par la mise en relation avec l'absolu renvoie - du moins ex negativo - à la possibilité d'un désarroi face à un monde dépourvu de sens.

24. Tugendhat, L'Homme égocentré, p. 3.

25. Ibid., p. 88. 
entre l'inclinaison à se disperser dans la recherche d'une multitude d'occupations pour chasser la pensée de la finitude de son existence d'un côté et le besoin opposé de se recueillir pour contempler le sens de son existence de l'autre; et c'est le besoin de recueillement qui est à l'origine, selon Tugendhat, de la religion et de la mystique. Pour lui, les deux sont à comprendre comme une sorte de " pas en arrière ${ }^{26}$ " qui permet à l'individu de surmonter l'égocentrisme qui caractérise l'homme habituellement et qui le rattache aux petits soucis quotidiens, tandis que dans la religion et dans la mystique, il parvient à se décentrer, à relativiser ses propres soucis en vue d'une perspective plus large pour ne plus autant se " prendre au sérieux ${ }^{27}$ ". Afın d'illustrer cette idée de décentrement, mais aussi pour préparer sa distinction entre religion et mystique, Tugendhat présente au lecteur l'exemple d'un juif orthodoxe et d'un bouddhiste Zen qui, respectivement, interprètent tout en vue de Dieu et en vue du vide universel. Dieu et le vide deviennent ainsi les points fixes autour desquels s'organise la multitude des événements et des choses au monde que perçoit l'individu. C'est dans le contexte de cette réflexion que Tugendhat introduit explicitement le concept de transcendance: "De ces deux points de référence, on peut dire qu'ils sont "transcendants" d'une manière ou d'une autre, qu'ils "ne sont pas de ce monde" 28 "; mais il va encore plus loin en formulant la position selon laquelle un point fixe permettant le recueillement doit, pour pouvoir remplir cette fonction, impérativement être "hors du monde", donc relever pour ainsi dire d'une transcendance verticale: "Ce que l'on peut en tout cas dire, c'est que la réponse ne peut être trouvée que dans quelque chose qui est "religieux au sens large", qui n'est "pas de ce monde" ${ }^{29}$."

Cette évocation de la notion de " religieux au sens large " nous renvoie à une conceptualisation particulière que Tugendhat introduit pour distinguer la religion de la mystique. Si Tugendhat voit donc une nécessité existentielle pour l'homme dans une perspective de transcendance, il tient à "tester" les différentes possibilités à la lumière de ce qu'il appelle, avec Nietzsche, l'» honnêteté intellectuelle ${ }^{30}$ ". Selon Tugendhat, seule la mystique peut se réclamer de cette honnêteté intellectuelle revendiquée, tandis que la religion, si elle remplit sa fonction en tant que point d'orientation, enfreint les critères que Tugendhat établit dans sa défınition de l'honnêteté intellectuelle. En effet, la religion est ce dont, selon Tugendhat, nous aurions à nous séparer si nous souhaitons garder un horizon de transcendance ouvert, mais sans entrer en conflit non seulement avec

26. Ibid., p. 97.

27. Ibid., p. 98. En allemand, Tugendhat utilise le verbe substantivé "Sichwichtignehmen ", ce qui correspond littéralement à " se prendre pour important ". L'adjectif " sérieux " me semble introduire ici une certaine ambiguïté dans la mesure où, en allemand, il existe une différence assez nette entre sich wichtig nehmen et sich ernst nehmen.

28. Ibid., p. 102.

29. Ibid., p. 103. Tugendhat propose deux autres options pensables: la possibilité de se recueillir autour d'une autre personne d'un côté et, de l'autre, de se recueillir autour de soi-même. Les deux seront par la suite écartés. Voir ibid., p.102-103.

30. Ibid., p. 57. 
les connaissances scientifiques et philosophiques actuelles, mais en principe avec l'ensemble de ce qu'on pourrait appeler les “narratifs” de la Modernité.

Comment Tugendhat justifie-t-il cette position? Comme il estime que le besoin de recueillement représente un élément essentiel de la structure anthropologique et ainsi un besoin légitime, la raison pour justifier le refus de la religion ne peut pas être la perspective de transcendance que la religion défend, puisqu'elle la partage avec la mystique. La position de Tugendhat se comprend principalement à travers la définition extrêmement restrictive que Tugendhat applique à la religion ${ }^{31}$ dans l'opposition à la mystique, définition qui s'appuie très strictement sur la tradition judéo-chrétienne (telle que Tugendhat l'interprète). Il comprend ainsi comme élément constitutif de la " religion " dans ce sens restrictif la croyance à des " êtres personnels supra-humains ${ }^{32}$ ". Il explique qu'on peut identifier, dans la croyance religieuse dans ce sens, une projection de ce qui nous semble souhaitable et ainsi trop clairement une sorte de reproduction des schémas humains à un niveau "surhumain", dans une sorte d'arrière-monde, comme le disait Nietzsche ${ }^{33}$. Tugendhat conclut son interprétation de la religion judéo-chrétienne ainsi conçue en postulant « que cette tradition n'est plus une possibilité $[\ldots]^{34}$, tandis que la mystique telle que je la défınis est une possibilité accessible à tous les hommes ${ }^{35}$ ".

31. Tugendhat parle également, comme nous l'avions vu, de la religion "au sens large” (ibid., p. 115) qui comprend la religion et la mystique. Sa "condamnation" de la religion concerne donc uniquement la religion "au sens restrictif".

32. Ibid., p. 105 (" übermenschliche personale Wesen ").

33. Voir ibid., p. 123-124: "Ce n'est pas seulement qu'on ne peut pas prouver l'existence de Dieu; l'idée même de l'existence des dieux ou de Dieu n'est pas compréhensible pour nous. Personne ne croit plus que les dieux vivent sur l'Olympe ou que Dieu est au-dessus des nuages; cela contredirait tout ce que nous savons. Dire en revanche que Dieu existe hors du temps et de l'espace nous oblige à avouer que nous sommes incapables de donner le moindre sens à l'idée qu'existe quelque chose qui ne soit pas localisable dans l'espace. À cela s'ajoute que la croyance en Dieu échoue sur la barrière de la conscience (Gewissen) intellectuelle dès qu'on se rend compte qu'elle est motivée par un souhait. " Si Tugendhat a certainement raison en ce qui concerne l'observation que ni les dieux grecs, ni un Dieu du type "vieil homme à la barbe blanche" ne peuvent satisfaire une conscience moderne, il me semble que cette réflexion demeure quelque peu caricaturale. Des convictions religieuses nettement plus nuancées sont tout à fait imaginables. Le problème de l'existence en dehors du temps et de l'espace obtient également une réponse assez péremptoire qui laisse transparaître l'influence analytique de la pensée de Tugendhat. Une perspective positiviste ne peut qu'aboutir à la conclusion qu'on ne puisse attacher aucun sens à un tel énoncé et il est certainement indubitable qu'un être au-delà du temps et de l'espace reste inaccessible à toute tentative de preuve scientifique (et même de saisie scientifique).

34. Tugendhat précise ici: "pour la perspective de la première personne " (ibid., p. 124.). Tugendhat distingue, dans l'accès à des phénomènes historico-culturels, la perspective de la troisième personne et celle de la première personne, la première représentant la perspective de la recherche scientifique et "objective", tandis que la seconde est celle de la philosophie, notamment quand il s'agit de poser la question de savoir " ce qu'il peut signifier pour nous et dans quelle mesure il existe des raisons et des contre-raisons d'emprunter soi-même ce chemin ou un chemin comparable " (ibid., p. 104-105). Il s'agit donc de la perspective de l'observateur d'un côté et de l'individu concret et existant - pour le dire avec Kierkegaard - de l'autre.

35. Ibid., p. 105. 
Comme Tugendhat écarte la possibilité d'une religiosité dans la tradition judéo-chrétienne, il propose une définition de la mystique qui s'inspire avant tout de la mystique telle qu'elle s'est développée en Inde et dans les pays de l'Asie de l'Est et qui correspond plus à son objectif de pouvoir rendre plausible la possibilité d'une perspective de transcendance que la mystique de la tradition chrétienne ${ }^{36}$. Tugendhat renvoie notamment à certaines traditions bouddhistes et taoïstes qui ne chercheraient pas, dans la méditation, le recueillement autour d'une " ultime réalité ${ }^{37}$ ", concept qui lui demeure suspect, comme les "arrière-mondes" de la tradition philosophique occidentale. Il préfère ainsi, pour sa définition de la mystique, le terme d'" univers " à celui d'" ultime réalité ". Selon sa défınition, la mystique consiste ainsi dans (1) la libération de tout " attachement volitif " qui caractérise habituellement notre rapport au monde; et ce (2) "face $\dot{a}[. .$.$] l'Univers { }^{38}$ ". Cette conception très large de la mystique offre à Tugendhat deux avantages: premièrement, elle permet de comprendre une grande diversité de formes de mystique, allant de la mystique orientale à la mystique judéo-chrétienne (auquel cas, Tugendhat le concède, on peut remplacer le terme d'univers par "Dieu”). En outre, cette défınition évite tout caractère ésotérique dans la mesure où elle peut se passer notamment de toute référence à l'idée d'ascèse souvent liée à certaines techniques de méditation, voire à la grâce divine. La mystique se présente ainsi, chez Tugendhat, comme une technique quelque part “démocratisée”, c'est-à-dire non réservée à des capacités extraordinaires ${ }^{39}$.

Nous n'avons pas ici la place de détailler l'analyse, tout à fait inspirante, des différents types de mystique que Tugendhat développe. Pour le présent contexte, il suffit de constater qu'il en ressort très clairement une volonté de favoriser une mystique dont l'orientation demeure dans le monde - comme cela serait, selon Tugendhat, le cas du taoïsme - et qui ne vise pas un dehors du monde, comme le bouddhisme. En outre, Tugendhat préfère à une mystique qui cherche l'unio mystica et dans laquelle la multitude des choses est ramenée à l'Un, une

36. Celle-ci consisterait davantage en l'expérience d'une unité immédiate avec Dieu et resterait, dans ce sens, attachée à la conceptualisation de divinités " personnalisées " (ibid., p. 116). Or, Tugendhat concède que certaines mystiques de la tradition judéo-chrétienne puissent, dans leur essence, rejoindre la mystique orientale.

37. Ibid., p. 106.

38. Ibid., p. 106 (c'est E.T. qui souligne).

39. Or, une "démocratisation" de la possibilité mystique telle que la propose Tugendhat ici, me semble impliquer un certain nombre de difficultés. On devrait notamment se poser la question de savoir d'où pourrait venir la capacité de faire taire la volonté si ce n'est par l'exercice spirituel dans la mesure où le décentrement de l'individu égocentré semble aller à l'encontre des structures anthropologiques actives habituellement. Il s'agirait, comme on pourrait dire en utilisant la terminologie heideggérienne, d'un arrachement au monde du "Man" dont on voit mal comment il pourrait se faire "spontanément". C'est d'autant plus problématique que la seule possibilité serait de dire que l'individu veuille effectuer un tel décentrement, auquel cas il ne parviendrait précisément pas à faire taire sa volonté. La grâce, l'autre possibilité envisageable, est également écartée par Tugendhat. Il me semble donc que cette démocratisation - sous la forme présentée demeure problématique sans, bien sûr, vouloir écarter par principe le caractère "démocratique" de l'expérience mystique. 
mystique dans laquelle la multitude des choses dans le temps et dans l'espace est conservée mais perçue dans son unité ${ }^{40}$. Dans cette préférence manifestée par Tugendhat pour le taoïsme, deux aspects importants entrent en ligne de compte, à savoir le statut qu'y occupe la négativité et tout particulièrement la souffrance, et puis l'orientation clairement pratique qui ne nécessite pas de constructions spéculatives sous-jacentes et peut donc se concevoir sans implications métaphysiques (dans le sens de la "mauvaise métaphysique"). Tugendhat voit ainsi dans le taoïsme un type de mystique qui parvient à proposer une réponse aux problèmes qui émergent en lien avec la question de savoir que ce nous voulons "au fond ", comme il l'avait formulé ${ }^{41}$. Tugendhat écarte donc une orientation religieuse "à l'ancienne" comme incompatible avec la conscience moderne (ou postmoderne), mais en revendiquant la possibilité d'une dimension transcendante. Plus encore: selon Tugendhat, il nous faut, en fin de compte, une perspective de transcendance, perspective seule à même de conduire, comme il le dit explicitement, à la " paix de l'âme ${ }^{42}$ ".

Un dernier aspect me semble important, notamment dans le contexte de la problématique esquissée dans l'introduction: Tugendhat construit son propos très clairement sur la conscience des carences et de la précarité de l'existence humaine au point que sa quête philosophique semble se nourrir principalement de l'inquiétude existentielle là où, chez Rentsch, c'était l'étonnement admiratif du monde qui prévalait. Or, Tugendhat conclut son essai sur l'homme égocentré et la mystique par un chapitre explicitement dédié à l'étonnement. On pourrait ainsi s'interroger sur la possibilité d'interpréter cette évolution réflexive partant du souci de la paix de l'âme et nous amenant vers la problématique de l'étonnement comme une sorte de parcours d'élévation à travers la réflexion philosophique qui est d'abord déclenchée par la prise de conscience de sa propre finitude et fragilité. Si celle-ci n'est pas refoulée dans la dispersion des préoccupations de la vie quotidienne et banale, elle permettrait ainsi de s'ouvrir au monde dans une perspective décentrée afın de le voir d'un regard différent qui, lui, peut être saisi à travers la notion d'étonnement philosophique (en accord avec Wittgenstein que Tugendhat cite dans ce contexte: "Qu'il est étrange que le monde existe ${ }^{43}$ ). Le décentrement mystique serait dans ce sens une attitude issue de l'étonnement philosophique, une attitude apaisante qui serait ainsi un remède aux angoisses existentielles ${ }^{44}$.

40. Voir ibid., p. 113sq.

41. Si Tugendhat souligne qu'il ne voit pas dans le taoïsme une "doctrine de salut " (ibid., p. 121), il suggère pourtant précisément le contraire. Voir ibid.: « Je ne veux pas préconiser le taoïsme comme doctrine de salut. Je crois simplement que le taoïsme a vu des problèmes que nous devrions aussi prendre au sérieux. "

42. Ibid., p. 125.

43. Ibid., p. 138.

44. Or, il s'agirait d'un remède dans le "bon" sens, c'est-à-dire dans le sens de la concentration et non pas de la dispersion; ce qui correspond structurellement à la distinction que l'on trouve dans les Pensées de Pascal entre un bonheur qui se fonde sur le divertissement et le "vrai" bonheur qui est celui des saints. 


\section{Conclusion}

Si nous essayons de résumer les réflexions précédentes, nous pouvons constater que c'est, tant pour Rentsch que pour Tugendhat, l'être-jeté dans l'existence en tant qu'être conscient qui est à l'origine de l'ouverture pour la perspective de transcendance. Comme l'homme ne peut pas vivre uniquement dans le présent, mais qu'il se voit toujours et inévitablement dans le contexte du passé qui a engendré le monde dans lequel l'homme est "jeté" et qui le précède structurellement (Thomas Rentsch parle de "Vorgängigkeit ${ }^{45}$ "), mais également dans la projection vers le futur, la structure même de son ek-sistence ouvre un horizon transcendant le présent. C'est la raison qui nous permet de - mais plus encore, si l'on suit Tugendhat et Rentsch, qui nous oblige à - nous transcender, que ce soit, comme c'est plutôt le cas chez Rentsch, dans la tradition chrétienne ou, comme le privilégie Tugendhat, dans une tradition à orientation orientale. Tant Rentsch que Tugendhat voient la nécessité d'un recueillement nous permettant de sortir de l'afflux incessant des "choses du monde". Dans leurs argumentations respectives en faveur d'une perspective de transcendance, il me semble d'ailleurs transparaître une condition cruciale permettant de pouvoir se construire " en tant qu'êtres libres et raisonnables ${ }^{46}$ ", chez Rentsch, ou dans l'optique d'une " paix de l'âme ", chez Tugendhat, qui va foncièrement à l'encontre du courant principal de la Modernité, plus explicitement chez Rentsch, mais de manière tout aussi présente chez Tugendhat. Cette argumentation va ainsi clairement à l'encontre de l'obsession de la maîtrise, du contrôle qui caractérise le monde contemporain, mais également à l'encontre du refus d'accepter la contingence et la finitude comme conditions inaccessibles à l'emprise de l'homme. Rentsch et Tugendhat s'accordent pour souligner que ce n'est pas par la tentative frénétique et vaine de manipuler les conditions qui nous fondent - dans une foi naïve et aveugle en l'avancée de la technologie notamment - que l'on atteint la paix de l'âme. Le rattachement à la perspective transcendante propose une approche radicalement différente qui consiste en la possibilité de sciemment lâcher prise. Un tel lâcher prise devient possible à travers un changement de perspective, à travers le décentrement, comme l'exprime Tugendhat, ou, chez Rentsch, à travers la possibilité de charger le monde fini d'un sens absolu grâce à la transcendance ${ }^{47}$.

Il est d'ailleurs significatif que tant Tugendhat que Rentsch dissocient explicitement la mystique (Tugendhat) et la transcendance (Rentsch) de toute réduction fonctionnaliste dans le sens de la spiritualité comme

45. Rentsch, Gott, p. 60.

46. Ibid., p. 82.

47. "Lâcher prise" et ainsi ne pas vouloir tout changer pour "rendre le monde tel qu'on le souhaiterait" ne veut d'ailleurs pas dire: tout accepter et s'adonner à un fatalisme passif. Tugendhat le souligne notamment dans la discussion du taoïsme en précisant que dans le taoïsme il y a la distinction entre " homme " et " ciel ", tout ce qui relève de l'homme peut faire l'objet d'un désir de changement, en revanche, ce qui relève du ciel - les conditions qui fondent notre existence doit être accepté. Or, la vraie sagesse est bien sûr de pouvoir savoir ce qui relève de l'un ou l'autre domaine (voir Tugendhat, L'Homme égocentré, p. 135-136). 
" Kontingenzbewältigungspraxis ${ }^{48}$ ", donc comme moyen afin de pouvoir accepter la contingence ${ }^{49}$. L'enjeu de la problématique ne peut pas être compris à travers une pensée pragmatico-fonctionnaliste qui, dans son orientation même, passe à côté de l'essence de ces perspectives de transcendance esquissées par Rentsch et par Tugendhat. D'une manière paradoxale, ce n'est donc qu'en laissant derrière elle une pensée fonctionnaliste que la perspective mystique - pour reprendre le terme de Tugendhat pour l'ensemble des phénomènes en question - peut remplir la fonction d'une telle Kontingenzbewältigung. Autrement dit, la mystique remplit cette fonction uniquement quand ce changement de perspective est enraciné dans une pensée qui ne pense plus dans de telles catégories. Il s'agit d'un changement de perspective si radical que toute pensée fonctionnaliste y est anéantie. Pour illustrer l'essence de ce changement fondamental de perspective, on peut citer une observation de maître Eckhart qui écrit: "Oui, si un homme s'abandonnait lui-même, quoi qu'il garde, richesse ou honneur, ou quoi que ce soit, il aurait abandonné toutes choses ${ }^{50}$. " Appliqué à la question de la fonction de la mystique comme Kontingenzbewältigung on pourrait résumer cette pensée de la manière suivante: c'est uniquement quand l'homme aura abandonné toutes choses dans l'expérience mystique et donc quand il aura abandonné toute pensée fondée sur des catégories fonctionnalistes et que la pensée fonctionnaliste aura perdu sa fonction que la mystique pourra fonctionner.

48. Rentsch, Gott, p. 37.

49. On peut tout de même poser la question de savoir si Tugendhat ne reprend pas une telle perspective malgré lui en parlant de la religion et du mysticisme comme de 'solutions' pour au problème de la contingence.

50. Cité dans Kurt Ruh, Initiation à Maître Eckhart. Théologien, prédicateur, mystique, Fribourg, Éditions universitaires Fribourg Suisse, 1997, p. 40. C'est dans le même sens que Schelling, en référence à Paul, Corinthiens 2 note: "Avoir comme si on n'avait pas: cela est en l'homme, cela est en Dieu le plus haut " (Friedrich Willhelm Schelling, cité dans Joseph Cohen: " Du Dieu à venir à l'ultime Dieu. De Schelling à Heidegger ", dans G. Bensussan et al., L'Héritage de Schelling. Interprétations aux XIX et $X X^{e}$ siècles, Freiburg, Karl Alber, 2015, p. 289-308, ici p. 300). 